\title{
Behaviour and the synapse
}

DOI:

10.1038/nrn1945

URLs

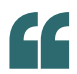

The ability

to measure

sensory

processing in

fully awake

mice opens

new avenues

of possibility

for unravelling

the true nature

of sensory

responses...
Sylvain Crochet and Carl Petersen from the Brain Mind Institute in Lausanne, Switzerland, have developed an impressive system for measuring cortical sensory neuron activity in awake mice. The report in Nature Neuroscience is the first to describe the membrane potential dynamics of single sensory neurons as they directly correlate to behaviour.

Neurons of the barrel cortex region are directly linked to the whiskers, and transmit whisker movement into synaptic signals. The team trimmed all the whiskers except one (the C2 whisker) of each mouse and, using a high-power intrinsic optical imaging approach, were able to identify the specific barrel cortex neuron responsible for the $\mathrm{C} 2$ signal. To investigate the synaptic events that are central to sensory processing, they made whole-cell patch-clamp recordings of the membrane potential of this barrel cortex neuron while the C2 whisker was either manually stimulated or quantified for movement during unstimulated behaviours.
The team first measured membrane potential variations when the mice were not moving their whiskers and then compared this to membrane potentials when the mice were whisking - moving their whiskers rapidly in an exploratory fashion. Whisker movement was filmed throughout in order to quantify movement and to correlate movement with membrane potential variation. They observed slow-frequency, large-amplitude membrane potential changes in barrel cortex neurons while whiskers were stationary, and this switched to high-frequency, small-amplitude changes during whisking.

The researchers next studied the sensory response elicited by manually stimulating the $\mathrm{C} 2$ whisker. To do this they glued a small metal particle to the $\mathrm{C} 2$ whisker and used a brief magnetic pulse to move the whisker. If the whiskers were stationary when the magnetic stimulation pulse was used, the neuron showed a large depolarization response. However, if the magnetic stimulation occurred during whisking the amplitude of response was decreased.
The measurements that Crochet and Peterson have managed to obtain show that membrane potential, and therefore sensory processing and perception, differs depending on whether mice are in a calm and stationary state or displaying exploratory whisker behaviour. Essentially, when mice are passive and not whisking, the same level of sensory input evokes a much greater response than when mice are active and exploratory. The ability to measure sensory processing in fully awake mice opens new avenues of possibility for unravelling the true nature of sensory responses and the effect of behaviour on sensory perception.

\section{Ruth Williams}

ORIGINAL RESEARCH PAPER Crochet, S. \& Peterson, C. H. Correlating whisker behavior with membrane potential in barrel cortex of awake mice. Nature Neurosci. 9, 608-610 (2006) 